\title{
Confronto sperimentale tra tecniche di misura della ventilazione naturale degli edifici
}

\section{An experimental comparison between different methods to measure building natural ventilation}

\section{Sommario}

Questo contributo è focalizzato sul confronto tra due metodi di misura per la caratterizzazione della ventilazione negli edifici: la misura della permeabilità all'aria (test di pressurizzazione) e la misura del tasso di ricambio d'aria (test di decadimento del gas tracciante). In particolare, nel lavoro vengono messe in risalto le criticità legate alla possibile correlazione tra dati forniti dai due diversi test. A tale scopo, è stata effettuata un'analisi sperimentale su un appartamento situato nel Centro-Sud Italia conducendo test di pressurizzazione e di decadimento del gas tracciante sia sull'intero appartamento che su singole zone. I risultati hanno dimostrato che il test di pressurizzazione condotto sull'intero appartamento non permette di identificare eventuali gradienti di permeabilità delle diverse zone. Inoltre, i test di decadimento del gas tracciante hanno evidenziato che la misura del ricambio d'aria dell'edificio è fortemente influenzata dalle condizioni climatiche esterne, per cui la stima di questa grandezza sulla base dei dati di permeabilità all'aria può comportare significative sovrastime del ricambio d'aria reale misurato mediante test di decadimento; in tal senso è fondamentale misurare la velocità e la direzione del vento "locale" contestualmente alle prove.

\section{Parole chiave:}

- Test di pressurizzazione

- Permeabilità all'aria

- Metodo del gas tracciante

- Tasso di ricambio d'aria

- Ventilazione

\section{Abstract}

The paper is focused on the comparison between two measurement methods typically adopted to characterize the building ventilation: the measurement of the air permeability (pressurization test) and the measurement of the actual air exchange rate (tracer gas decay test). In particular, the criticalities related to the correlation of air permeability and air exchange rate data are highlighted and stressed. To this aim, an experimental analysis has been carried out in a dwelling located in the Central-South Italy performing air permeability and air exchange rate measurements both in the whole dwelling and in parts of it. The results demonstrate that the pressurization test carried out on the whole dwelling does not allow to identify possible air permeability gradients within the envelope. Moreover, the measurement of the actual air exchange rate is strongly influenced by the outdoor microclimatic conditions; thus, the estimate of the air exchange rate on the basis of the air permeability data could lead to significative overestimates with respect to the actual air exchange rates. Therefore, a proper measurement of the local wind velocity and direction is mandatory.

\section{Keywords:}

- Blower door test

- Air permeability

- Tracer gas decay method

- Air exchange rate

- Ventilation 


\section{Introduzione}

L'obbligo di realizzare edifici a energia quasi zero, gli NZEB (Attia et al., 2018; D'Agostino et al., 2019), costringe a coniugare e ottimizzare due esigenze spesso antitetiche: la prestazione energetica e la qualità dell'ambiente interno, la IEQ, che comprende tra l'altro gli aspetti della progettazione legati al comfort termico e alla qualità dell'aria indoor, strettamente connessi ai consumi di energia, il cui conseguimento rischia quindi di compromettere il raggiungimento degli obiettivi di riduzione del fabbisogno di energia tipico degli NZEB (D'Agostino et al., 2018; Guyot et al., 2018). In particolare, nel caso degli NZEB, caratterizzati da limitate perdite per trasmissione e da una elevata efficienza degli impianti (Stabile et al., 2019), il contributo delle perdite di energia per ventilazione, spesso ritenuto trascurabile negli edifici tradizionali, può diventare preponderante.

Per verificare che le prestazioni energetiche di un edificio, nuovo o esistente, siano effettivamente quelle richieste, è sempre più importante caratterizzarlo in termini di permeabilità all'aria e determinarne l'effettivo ricambio d'aria.

La permeabilità all'aria viene misurata con la tecnica della pressurizzazione, cioè con il Blower Door Test, BDT, definita dalla norma UNI EN ISO 9972 (UNI, 2015), che permette di valutare l'ermeticità dell'edificio quando sottoposto a differenze di pressioni interno-esterno maggiori di quelle dovute alle forzanti climatiche esterne. In particolare, il BDT è basato sulla pressurizzazione o depressurizzazione dell'edificio o di una singola stanza e sulla simultanea misura della portata che, sotto una differenza di pressione imposta tra ambiente interno ed esterno $(\Delta p)$, attraversa l'edificio. La relazione tra la portata d'aria, $q_{\text {env }}$ in $\mathrm{m}^{3} / \mathrm{h}$, e la differenza di pressione, $\Delta p$ in $\mathrm{Pa}$, è data dall'equazione

$$
q_{\mathrm{env}}=C_{\mathrm{env}}(\Delta p)^{n}
$$

dove $n$ e $C_{\text {env }}$ sono parametri stimati secondo una semplice regressione lineare indicata dalla norma.

II metodo del decadimento di un gas tracciante, normato dalla ISO 12569 (ISO, 2017) permette di misurare il ricambio d'aria in funzione delle condizioni climatiche effettive del periodo di misura. Alcuni ricercatori hanno cercato di correlare le informazioni fornite dalle due tecniche in modo da poter stimare con buona approssimazione il ricambio d'aria reale di un edificio opportunamente caratterizzato in termini di permeabilità all'aria (Persily et al., 1983; Sundell et al., 2011; Nabinger et al., 2011; d'Ambrosio Alfano et al., 2012; d'Ambrosio Alfano et al., 2016; Stabile et al., 2016). La tecnica BDT, infatti, pur essendo molto ripetibile non fornisce direttamente il ricambio d'aria.

Di seguito vengono analizzati e discussi i due metodi e vengono illustrate le difficoltà nel correlare i dati di permeabilità a quelli di ricambio d'aria effettivi utilizzando i risultati di una campagna sperimentale intensiva effettuata in un appartamento residenziale, sia sull'intero appartamento che sui singoli locali.

\section{Materiali e metodi}

Lo studio sperimentale è stato condotto in un appartamento residenziale tipico degli anni '70 situato al secondo piano di un edificio del centro storico del comune di Frosinone. L'edificio presenta una facciata, esposta a Sud-Ovest, prospiciente un'ampia piazza, e una facciata più piccola e riparata, esposta a Nord-Est, come mostrato in Figura 1.

L'appartamento, la cui pianta è riportata in Figura 2, è costituito da nove zone di diverse dimensioni per una superficie netta totale del pavimento di $135 \mathrm{~m}^{2}$ e un volume interno netto totale di $378 \mathrm{~m}^{3}$. La porta di ingresso e le sette finestre sono sprovviste di sigillatura; nello specifico, gli infissi, con telaio in legno a singolo vetro, rientrano nella classe 1 (livello minimo) di permeabilità all'aria secondo

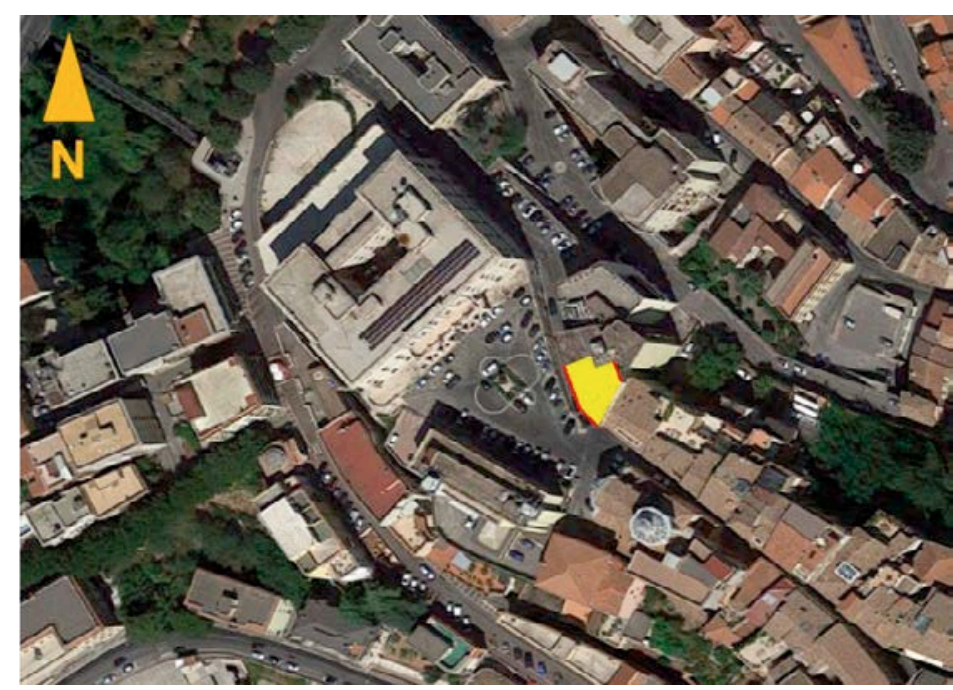

Figura 1 - Posizione dell'edificio oggetto di studio (in giallo) nel centro storico di Frosinone. Nell'immagine sono evidenziate (in rosso) le facciate dell'edificio prospicienti l'ambiente esterno Figure 1 - Location of the building under investigation (in yellow) in the city. In the figure the building facades are also highlighted (in red)

la classificazione della norma UNI EN 12207 (UNI, 2017). La muratura esterna dell'edificio è costituita da mattoni forati con intercapedine d'aria (spessore totale di $45 \mathrm{~cm}$ ). L'appartamento non è dotato di sistemi di ventilazione meccanica, per cui i ricambi d'aria sono demandati alla ventilazione naturale e, quindi, sono strettamente connessi alla permeabilità intrinseca dell'appartamento.

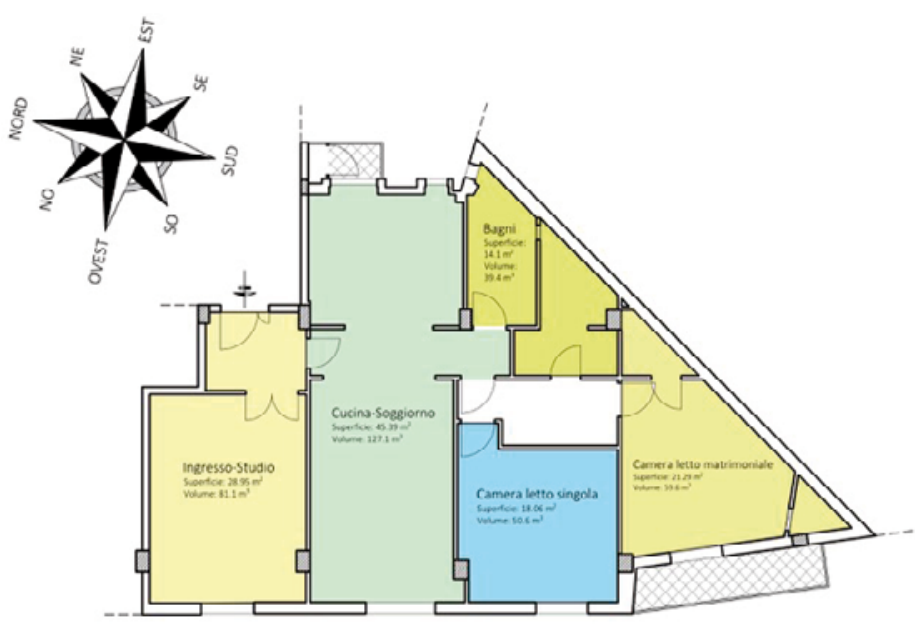

Figura 2 - Planimetria dell'appartamento oggetto dello studio e identificazione delle zone/locali oggetto di misure BDT

Figure 2 - Plant of the dwelling under investigation: identification of zones/rooms where the pressurization tests were performed

Le misure e i successivi confronti hanno riguardato l'intero appartamento e cinque singoli locali in comunicazione con l'ambiente esterno. In particolare, sono stati misurati:

- la permeabilità all'aria delle singole stanze mediante tecnica di pressurizzazione;

- la permeabilità all'aria dell'intero appartamento mediante tecnica di pressurizzazione;

- il ricambio d'aria delle singole stanze mediante tecnica di decadimento del gas tracciante;

- il ricambio d'aria dell'intero appartamento mediante tecnica di decadimento del gas tracciante.

La permeabilità all'aria dell'appartamento e dei singoli locali è stata misurata con il metodo della pressurizzazione secondo le indicazioni della norma UNI 9972 (UNI, 2015), utilizzando il Metodo 1, "test di un edificio in uso", che permette di condurre misure di permeabilità 
dell'edificio nelle reali condizioni di utilizzo dell'edificio, cioè senza sigillare porte e finestre.

Le misure sono state condotte utilizzando un ventilatore a portata controllata, in grado di indurre differenze di pressione positive o negative, un telaio estensibile e un telo di nylon per garantire la sigillatura del locale oggetto della misura, un sistema di controllo della pressurizzazione/depressurizzazione per la regolazione della portata e del verso del flusso in grado di imporre un $\Delta p$ desiderato tra interno ed esterno, due elementi primari per la misura della portata d'aria, un micro-manometro digitale per la misura del $\Delta p$ tra interno ed esterno, una sonda multifunzione per la misura di temperatura, pressione e umidità relativa interne ed esterne utili alla normalizzazione delle portate. II ricambio d'aria a $50 \mathrm{~Pa}\left(n_{50}\right)$ come rapporto tra la portata a $50 \mathrm{~Pa}\left(q_{50}\right)$ e il volume dell'appartamento o del locale è stato calcolato con la (1). Sulla base del valore di $n_{50}$ è stato anche stimato il ricambio d'aria in condizioni naturali $(n)$ dividendo $n_{50}$ per un fattore $N$, che, sulla base di quanto riportato in letteratura e validato con buona approssimazione per edifici del Nord America (Persily et al., 1983; Sundell et al., 2011), è stato posto pari a 20.

II ricambio d'aria nell'appartamento e nei singoli locali è stato misurato mediante il test di decadimento della $\mathrm{CO}_{2}$ secondo l'approccio "single zone", indicato dalla norma ISO 12569 (ISO, 2017), che consente di stimare il ricambio d'aria sulla base della velocità di riduzione della concentrazione della $\mathrm{CO}_{2}$ nel locale in esame. Come prescritto dalla norma, è stata immessa nel locale $\mathrm{CO}_{2}$ la cui concentrazione, uniformata azionando dei ventilatori, è stata misurata con una sonda di $\mathrm{CO}_{2}$ operante secondo il principio di misura NDIR. Durante le prove, le porte e le finestre dell'appartamento e dei locali sono state tenute chiuse, così da essere certi che il ricambio d'aria fosse funzione solo della permeabilità dell'edificio e del $\Delta p$ tra interno ed esterno dovuto alle forzanti meteo-climatiche. Infatti, come accennato, a differenza del BDT i risultati ottenuti con il metodo del decadimento sono funzione delle condizioni meteo-climatiche esterne e, quindi, per definizione, non ripetibili. II ricambio d'aria è stato stimato con la relazione:

$$
n=(1 / \Delta t) \ln \left[\left(C_{1}-C_{0}\right) /\left(C_{2}-C_{0}\right)\right.
$$

dove $C_{1}, C_{2}$ e $C_{0}$ sono, rispettivamente, le concentrazioni di picco, finale ed esterna di $\mathrm{CO}_{2}$ e $\Delta t$ è l'intervallo di tempo tra $C_{1}$ e $C_{2}$.

Le misure mediante tecnica di pressurizzazione sono state condotte una sola volta per ciascun locale individuato, data l'elevata ripetibilità del metodo, mentre quelle mediante il metodo del decadimento dei gas traccianti sono state ripetute diverse volte in ragione di quanto detto sulla dipendenza dalle condizioni climatiche esterne.

\section{Risultati e discussione}

In Tabella 1 sono riportati i risultati delle misure effettuate con il metodo BDT in termini di ricambio d'aria a $50 \mathrm{~Pa}, n_{50}$, per i singoli locali dell'appartamento in esame e per l'intero appartamento e i valori stimati dei ricambi naturali, $n$, espressi sia come mediana delle diverse misure che come intervallo minimo-massimo dei dati misurati.

I valori di $n_{50}$ relativi ai singoli locali, che variano da 13,6 a 15,4 h-1, evidenziano una sensibile disuniformità del comportamento dell'appartamento in termini di permeabilità all'aria, infatti il valore di $n_{50}$ sull'intero appartamento è pari a circa $12 \mathrm{~h}^{-1}$, ossia inferiore al valore stimato calcolando la media pesata sui volumi delle permeabilità dei singoli locali, pari a 14,9 h-1. Tale scostamento può essere imputato a flussi interstiziali tra i locali adiacenti non apprezzabili con il metodo BDT applicato all'intero appartamento. Ad ogni modo, una più dettagliata analisi della differenza tra i due metodi necessita un'attenta analisi delle incertezze delle due misure, il che rappresenta un obiettivo futuro dello studio.
Per quanto riguarda i valori di $n$ misurati mediante test di decadimento della $\mathrm{CO}_{2}$, in Tabella 1, è evidente una loro elevata variabilità attribuibile, in buona parte, alle diverse condizioni climatiche esterne. Inoltre, il rapporto tra i valori $n_{50}$ del BDT ed i valori mediani di $n$ del test di decadimento per i singoli locali investigati risulta pari a circa 41 per la zona 1 (ingresso-studio), circa 31 per la zona 2 (cucina-soggiorno), circa 51 per la zona 5 (camera da letto matrimoniale) e circa 76 per l'intero appartamento. Ė opportuno sottolineare che tali rapporti sono decisamente maggiori del valore di riferimento, come detto posto pari a 20. Inoltre, se si considerano i valori minimi e massimi di $n$ in Tabella, il rapporto $n_{50} / n$ varia tra 16 e 100, che è anch'esso al di sopra dell'intervallo 10-30 indicato dalla letteratura (Persily et al., 1983; Sundell et al., 2011). La variabilità del rapporto $n_{50} /$ nè messa in luce in Figura 3, dove sono riportati i rapporti ottenuti in tutte le misure condotte nei locali dell'appartamento in ragione della componente normale della velocità del vento $(u)$ misurata dalla centralina ARPA di zona. La Figura 3 mostra una forte dipendenza dalla forzante esterna: infatti, all'aumentare della componente $u$ della velocità incidente sulle finestre dell'appartamento si nota una sensibile riduzione del rapporto $n_{50} / n$ e della dispersione dei dati misurati. Dal punto di vista fisico, questo fenomeno è attribuibile alla circostanza che una minore forzante esterna, cioè una ridotta velocità del vento, comporta un minore $\Delta p$ e, quindi, un minore ricambio d'aria. L'elevata dipendenza del rapporto $n_{50} / n$ dalla velocità del vento determina l'impossibilità di definire un unico parametro $N$ convenzionale valido indipendentemente dalle condizioni climatiche esterne. Va inoltre osservato, come già evidenziato in precedenza, che anche in presenza di elevate velocità del vento il rapporto $N$ resta mediamente maggiore di 20. È opportuno puntualizzare che il rapporto $n_{50} / n$ è fortemente influenzato dall'intensità e dalla direzione del vento locali, ovvero nell'immediata prossimità dell'edificio, che possono variare in funzione dell'orientamento dell'edificio, ma anche di ostacoli quali altri edifici e alberi, della presenza di street canyon, ad esempio strade e canaloni, e dell'orografia locale e, quindi, di parametri difficilmente identificabili dalla centralina meteo di zona e tutto ciò potrebbe spiegare, in parte, l'elevata dispersione dei risultati ottenuti.

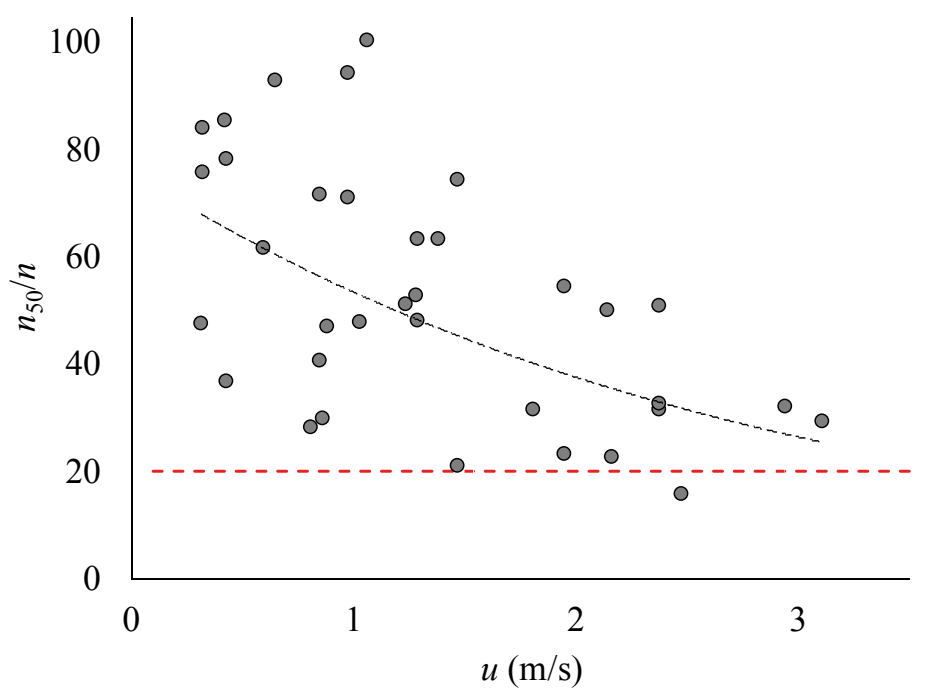

Figura 3 - Rapporto n50/n misurato nei diversi locali dell'appartamento in esame in funzione della componente normale della velocità del vento $(\mathrm{u})$. In rosso è riportato il parametro $\mathrm{N}$ $=\mathbf{2 0}$ suggerito dalla letteratura scientifica del settore

Figure $3-n 50 / n$ ratios measured in the different zones as a function of the normal component of the wind velocity $(u)$. The $N=20$ line (suggested by the scientific literature) is also highlighted 
Tabella 1 - Valori misurati di $\mathrm{n}_{50}$ mediante Blower Door Test sui singoli locali e sull'intero edificio, stima del ricambio d'aria a partire dai dati di $\mathrm{n}_{50}$ e valori misurati di ricambio d'aria secondo la tecnica del decadimento del gas tracciante $\left(\mathrm{CO}_{2}\right)$

Table I $-n_{50}$ values measured through the pressurization test performed in the rooms and in the whole dwelling, air exchange rates estimated from the $n_{50}$ values, and actual air exchange rates measured according to the tracer gas decay method $\left(\mathrm{CO}_{2}\right)$

\begin{tabular}{|c|c|c|c|c|c|}
\hline \multirow{3}{*}{ Zona } & \multicolumn{2}{|c|}{ BDT } & \multicolumn{3}{|c|}{ Test decadimento della $\mathrm{CO}_{2}$} \\
\hline & \multirow{2}{*}{$n_{50}\left(\mathrm{~h}^{-1}\right)$} & \multirow{2}{*}{$n=n_{50} / N\left(\mathrm{~h}^{-1}\right)$} & \multicolumn{2}{|c|}{$n\left(\mathrm{~h}^{-1}\right)$} & \multirow{2}{*}{$\begin{array}{r}\text { stimato } \\
\text { mediana }\end{array}$} \\
\hline & & & mediana & intervallo & \\
\hline 1) Ingresso-Studio & 13.8 & 0.69 & 0.34 & $0.14-0.70$ & 40.6 \\
\hline 2) Cucina-Soggiorno & 15.9 & 0.79 & 0.52 & $0.22-0.90$ & 30.5 \\
\hline 3) Bagni & 14.7 & 0.74 & - & - & - \\
\hline 4) Camera letto singola & 13.6 & 0.68 & - & - & - \\
\hline 5) Camera da letto matrimoniale & 15.4 & 0.77 & 0.30 & $0.16-0.96$ & 51.2 \\
\hline \multicolumn{6}{|l|}{ Appartamento } \\
\hline Media stimata delle singole zone & 14.9 & 0.75 & - & - & - \\
\hline Misura sull'intero appartamento & 12.2 & 0.61 & 0.16 & $0.14-0.33$ & 76.1 \\
\hline
\end{tabular}

\section{CONCLUSIONI}

La campagna sperimentale intensiva condotta su un appartamento ad uso privato ha consentito di valutare vantaggi e svantaggi delle due diverse tecniche di misura della permeabilità di un edificio nonché di evidenziare alcune criticità nella misura della ventilazione naturale degli edifici. In particolare, sono state condotte misure di permeabilità all'aria, secondo la tecnica di pressurizzazione (Blower Door Test), e di ricambio d'aria, secondo la tecnica di decadimento della $\mathrm{CO}_{2}$.

I risultati della sperimentazione hanno evidenziato che:

- la misura con tecnica BDT condotta nell'intero appartamento non permette di identificare eventuali gradienti di permeabilità dei diversi locali/zone dell'appartamento;

- la misura con tecnica BDT nei singoli locali rischia di sovrastimare la permeabilità a causa della permeabilità interna tra i diversi ambienti;

- la stima del ricambio d'aria a partire dal valore di $n_{50}$ fornito dal
BDT, pratica utilizzata in letteratura, può condurre a importanti sovrastime dell'effettivo ricambio d'aria naturale $n$, anche perché i valori convenzionali del ricambio d'aria disponibili in letteratura sono per lo più riferiti a condizioni climatiche e soluzioni costruttive del Nord America;

- la misura del ricambio d'aria è fortemente influenzata dalle condizioni climatiche esterne; in tal senso è fondamentale misurare, contestualmente alle prove, la velocità e direzione del vento "locale".

Sviluppi futuri di questo studio dovranno contemplare un'accurata stima dell'incertezza delle due tecniche di misura considerate e un'analisi delle possibili disuniformità spaziali durante la misura del reale ricambio naturale in grandi locali, ad esempio nell'intero appartamento con la tecnica del decadimento del gas tracciante così da capire se i risultati sono influenzati dalla scelta del punto di posizionamento della sonda nelle prove effettuate secondo il metodo "single zone".

\section{CONFLITTO DI INTERESSE}

Gli autori dichiarano che non esistono conflitti di interesse di ordine economico o di altro tipo sull'articolo presentato.

\section{FINANZIAMENTI ALLO STUDIO}

Questi risultati sono stati ottenuti nell'ambito della ricerca o relativa al progetto PRIN "Riqualificazione del parco edilizio esistente in ottica NZEB/Renovation of existing buildings in NZEB vision (nearly Zero Energy Buildings)" finanziato dal MIUR (Grant number 2015S7E247_002).

\section{BIBLIOGRAFIA}

Attia S., Eleftheriou P., Xeni F., Morlot R., Ménézo C., Kostopoulos V. et al. Overview and future challenges of nearly zero energy buildings (nZEB) design in Southern Europe. Energy and Buildings 2018; 155:439-458.

D'Agostino D., Mazzarella L. Data on energy consumption and Nearly zero energy buildings (NZEBs) in Europe, Data in Brief 2018; 21:2470-2474.

D'Agostino D., Mazzarella L. What is a Nearly zero energy building? Overview, implementation and comparison of definitions. Journal of Building Engineering 2019: 21:200-212.

d'Ambrosio Alfano F.R., Dell'Isola M., Tassini F., Ficco G. Experimental analysis of air tightness in Mediterranean buildings using the fan pressurization method. Building and Environment 2012; 53:16-25.

d'Ambrosio Alfano F.R., Dell'Isola M., Ficco G., Palella B.I., Riccio G. Experimental air-tightness analysis in Mediterranean buildings after windows retrofit. Sustainability 2016; 8.

Guyot G., Sherman M.H., Walker I.S. Smart ventilation energy and indoor air quality performance in residential buildings: A review. Energy and Buildings 2018; 165:416-430

ISO, 2017. Thermal performance of buildings and materials - Determination of specific airflow rate in buildings - Tracer gas dilution method., ISO 12569.
Nabinger S., Persily A. Impacts of airtightening retrofits on ventilation rates and energy consumption in a manufactured home. Energy and Buildings 2011; 43:3059-3067.

Persily A.K., Linteris G.T. A comparison of measured and predicted infiltration rates, ASHRAE Transactions 1983; 89.

Stabile L., Dell'Isola M., Frattolillo A., Massimo A., Russi A. Effect of natural ventilation and manual airing on indoor air quality in naturally ventilated Italian classrooms. Building and Environment 2016; 98:180-189.

Stabile L., Buonanno G., Frattolillo A., Dell'Isola M. The effect of the ventilation retrofit in a school on $\mathrm{CO}_{2}$, airborne particles, and energy consumptions. Building and Environment 2019; 156:1-11.

Sundell J., Levin H., NazaroffW.W., Cain W.S., Fisk W.J., Grimsrud D.T. et al. Ventilation rates and health: multidisciplinary review of the scientific literature. Indoor Air 2011; 21:191-204.

UNI. 2015. Thermal performance of buildings - Determination of air permeability of buildings - Fan pressurization method. Norma UNI 9972. Milano: Ente Italiano di Normazione.

UNI. 2017. Finestre e porte - Permeabilità all'aria - Classificazione. Norma UNI EN 12207. Milano: Ente Italiano di Normazione. 